\title{
Sensor Network Design and Implementation for Health Telecare and Diagnosis Assistance Applications
}

\author{
Ming-Hui Jin ${ }^{1}$, Ren-Guey Lee ${ }^{2}$, Cheng-Yan Kao ${ }^{3}$,You-Rui Wu ${ }^{4}$,D. Frank Hsu ${ }^{5}$, Tse-Ping Dong ${ }^{6}$, and Kuan-Tsae Huang ${ }^{7}$ \\ ${ }_{1,3,4}$ Department of Computer Science and Information Engineering, National Taiwan University, Taipei, Taiwan \\ ${ }^{2}$ Department of Electronic Engineering, National Taipei University of Technology, Taipei, Taiwan \\ ${ }^{5}$ Dep. of Computer and Information Sciences, Fordham University, LL813, New York, NY 10023, U. S. \\ ${ }^{6}$ Graduate Institute of Business Administration, National Taiwan University, Taipei, Taiwan \\ ${ }^{7}$ National Taiwan Normal University, Taipei, Taiwan
}

\begin{abstract}
Attempts to develop a ubiquitous health care monitoring system arisen from the need of automatic real-time medical services for emergent diseases. Besides, the physiological statuses gathered and maintained by this system are very helpful for diagnosis and early warning. To improve the medical services and diagnosis accuracy, a Wireless Health $\boldsymbol{A}$ dvanced Mobile Bio-diagnostic System (abbreviated as WHAM-BioS) is proposed. This study focuses on network/communication technology in the WHAM-BioS and proposes a novel clustered sensor network (CSN) architecture for long-term periodical telecare applications. In the proposed CSN architecture, most network functions are concentrated in a special purpose device called the human body gateway (HBG). The sensor nodes focus on detecting and reporting their detection results to their HBG. To reduce the design complexity and the implementation cost for the sensor nodes, the proposed architecture proposed several protocols to help each HBG to provide a contention free environment for their sensor nodes. The contention free environment significantly reduces the power consumption in data retransmission. Besides, to further reduce the power consumption of the sensor nodes, this study also proposes a power saving mechanism which reduces the power consumption in idle listening. Based on the proposed network architecture and protocols, a prototype system is implemented.
\end{abstract}

\section{INTRODUCTION}

Considering the cutting-edge technology and scientific development, biotech, information technology and communication technology are three mainstream industries in this century. The semiconductor giant, Motorola, announced it would invest 5 billion dollars in micro-electro-mechanical systems (MEMS) and wireless communication to keep its competitive advantage. The eSensor ${ }^{\mathrm{TM}}$ DNA detection system and corresponding Reader is the representative result in its Life Science Department. In all these research topics, the system integration and nanotechnology breakthrough form the foundations. In other words, cross-disciplinary integration is must in all these efforts.

Following this new wave of industry revolution, Taiwan's Ministry of Economic Affaires supports National Taiwan University (NTU) Nano-BioMEMS Group to develop a "Wireless Health Advanced Monitoring Bio-Diagnosis System (WHAM-BioS)" that encompasses the integration of biotechnology, information technology, and network/communications, Nanotechnology/MEMS, etc. With long-term home care as the primary market, the full project proposes to perform real-time C-Reaction Protein (CRP) detection in blood serum. Using a miniature RF system, which can then be linked into the GSM/GPRS network for bio-informatics analysis, will then transmit the data obtained forward.

This paper focuses on the network design and implementation for the WHAM-BioS. The network design and implementation for the WHAM-BioS brings numerous challenges. The main challenge arises from power saving. Since all the biosensors, RF and other elements of each sensor node contest the scarce power resources, the elements of any sensor node are required to minimize their power consumption. Therefore, power consumption minimization is the goal of network design for the sensor node implementation.

Since the power consumption of data transmission increases significantly with the communication ranges [1], short range transmission becomes crucial for power consumption minimization. This implies that reporting the detection results from the sensor nodes to the Internet access points such as Wireless LAN access points or GPRS basestations is impractical. Therefore, providing a special portable device with more power resources for forwarding the detections results would be a practical approach. In this paper, the special portable device for forwarding the detection results from sensor nodes to the Internet access points is called the Human Body Gateway (HBG).

Although the approach above is practical, it conceals some problem issues. First, the packets which are reported from the sensor nodes to the HBG may collide. Second, the transmission from the sensor nodes in one person to their HBG may interfere the transmission in another person nearby. These two events either require the sensor nodes to retransmit their detection results or eliminate the detection results. Both the two events waste the scarce power resources of the corresponding sensor nodes.

Most wireless sensor networks such as the WINS[2], the PicoRadio[3] and the AMPS[4] base their design on an ad hoc (multi-hop) network technology $[5,6,7]$ that focus on organizing and maintaining a network formed by a group of moving objects with a communication device in an area with no fixed base stations or access points. Although ad hoc network technologies are capable of solving the MAC [8] issues above, the design and implementation of sensor networks for long-term health telecare applications can be furthered simplified to reduce power consumption and overhead.

We proposed the HSN architecture in [9] for sensor networks with immobile sensor nodes. Although the sensor node - LCC relationships in the HSN is similar to the sensor 
nodes - HBG relationships in human body sensor networks, the HSN architecture can not be applied in the long-term health telecare applications directly because the HSN architecture focus on the applications in which all the senor nodes are immobile. Besides, the self-organization protocol [5] for constructing the sensor node - LCC relationships should be removed from the HSN architecture since the relationship can be manually set and auto-organization may bring incorrect relationship construction. Therefore, in this study, we appropriately modify the HSN architecture and then proposed a novel sensor network architecture called the clustered senor network (CSN) for long-term health telecare applications.

This paper is organized as follows. Section 2 presents the CSN architecture, the power saving mechanism and the inner cluster communication protocols. Section 3 presents the inter cluster communication protocols. Based on the proposed architecture, a sensor network system prototype is implemented and evaluated in Section 4. Some important issues are discussed in Section 5. The conclusions and future works are drawn in Section 6.

\section{NETWORK ARCHITECTURE AND PROTOCOLS}

\subsection{The Network Architecture}

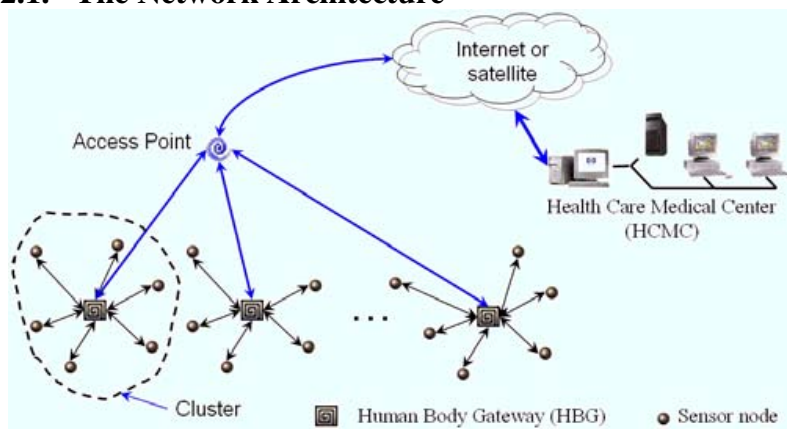

Fig. 1 The cluster sensor network architecture

Fig. 1 shows the proposed network architecture. In this architecture, the network is partitioned into several clusters each one of which maintains the status of a human body. Each cluster contains several sensor nodes and a Human Body Gateway (HBG). A sensor node has capability to detect and then reports the detection results to its HBG. The detection results are then forwarded to the nearby access points directly. The access points then forward the detection results to the Health Care Medical Center (HCMC) Internet or satellite.

In this architecture, all the sensor nodes maintain no network information. Whenever a sensor node is installed in a human body, the installer manually constructs the master-slave relationship between the sensor node and the HBG. Whenever the sensor node joined the network, it does nothing unless it receives an instruction from its HBG. The HBG applies the polling protocol to avoid the collision between the packets from different sensor nodes in the same cluster. This centralized communication protocol shifts most network maintenance tasks from the sensor node to the HBG and hence significantly minimizes the design and implementation complexity of the communication module of the sensor nodes.

Although the sensor nodes possess poor mobility functions, however, the proposed protocols provide group mobility functions. As long as the sensor nodes keep connection to their HBG, they can move with their HBG without any injury. Therefore, this architecture is well appropriate for sensor networks with group moving sensors.

\subsection{The Power Saving Mechanism}

Power resource is precious for sensor nodes in many applications [5, 6, 7]. To further reduce the power consumption for the sensor nodes, the concept of cluster node is introduced. Fig. 2 shows the life cycle of a cluster node.



Fig. 2. The life cycle of a cluster node.

Whenever a HBG is turned on, the cluster node is in the initiation stage. It changes its stage to the operation stage once it completes the initialization tasks. In the operation stage, the cluster node may be in active mode or idle mode. A HBG is allowed to communicate with its sensor nodes only if the corresponding cluster node is in the active mode. This implies that the sensor nodes can largely reduce their power consumptions by turning off their antennas when their cluster nodes are in idle mode. Whenever the user turns off the HBG or the power resource of the HBG is less then a predetermined threshold, the corresponding cluster node changes its stage to the hibernation stage. The cluster node may revive again after someone turns on the HBG.

Although this mechanism significantly reduces the power consumption of all the sensor nodes in the operation stage, it also brings two new problems below.

1. Once a sensor node turns off its antenna, its HBG has no way to communicate with it. Therefore, a protocol is necessary for correctly setting the alarm clock of the sensor nodes.

2. If two adjacent cluster nodes are in active mode simultaneously, the communications in one cluster may interferes with the communications in another. This implies that adjacent cluster nodes should not be active simultaneously. Therefore, a protocol for solving the simultaneous activity problem is crucial for network maintenance.

For convenient, the protocols for solving the first problem are called the inner cluster communication protocols and the protocols for solving the second problem are called the inter cluster communication protocols.

\subsection{The Inner Cluster Communication Protocols}

The power saving mechanism introduces the concept of detection cycle which can be partitioned into active interval and idle interval. In Fig. 3, a cluster node becomes active for $t_{A}$ milliseconds and become idle for $t_{I}$ milliseconds in every detection cycle.

\begin{tabular}{|c|c|c|c|c|c|}
\hline $\begin{array}{l}\text { Active } \\
\text { Interval }\end{array}$ & $\begin{array}{c}\text { Idle } \\
\text { Interval }\end{array}$ & $\begin{array}{l}\text { Active } \\
\text { Interval }\end{array}$ & $\begin{array}{c}\text { Idle } \\
\text { Interval }\end{array}$ & $\begin{array}{l}\text { Active } \\
\text { Interval }\end{array}$ & $\begin{array}{c}\text { Idle } \\
\text { Interval }\end{array}$ \\
\hline$t_{A}$ & $t_{I}$ & $t_{A}$ & $t_{I}$ & $\overbrace{t_{A}}$ & $\overbrace{t_{I}}$ \\
\hline
\end{tabular}

Fig. 3. The detection cycle of a cluster node 

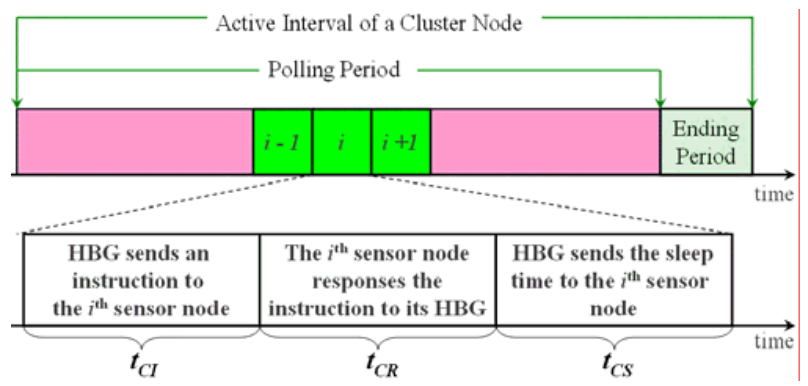

Fig. 4. The compositions of an active interval

The first inner cluster communication protocol specifies that each active interval should be partitioned into two periods as Fig. 4. In the polling period, the HBG communicates with all it sensor nodes one by one. Each communication consists of three steps. In the first step the HBG sends an instruction to the sensor node. Once the sensor node received the instruction, it performs the corresponding procedure to response the instruction in the second step. Finally, the HBG sends the sleep time to the sensor node. Once the sensor node receives the sleep time from its HBG, it applies the sleep time to set a counter for waking up its antenna and then turns off its antenna. In this paper, the times for the three steps are denoted as $t_{C I}, t_{C R}$ and $t_{C S}$, respectively. Besides, this protocol also requires that the values of $t_{C I}$ and $t_{C S}$ are constants and are well known by all the members in the same cluster. Based to this protocol, each HBG can easily calculate the value of $t_{A}$ of its cluster node. Furthermore, once the HBG determines the value of $t_{I}$, the HBG can immediately calculate the sleep time of its sensor nodes.

The HBG broadcasts the value of $t_{I}$ to all its sensor nodes. The ending period is very useful to the sensor nodes which have no idea about their sleep time. The second inner communication protocol provides a resynchronization mechanism for the sensor nodes which have no idea about their sleep time.

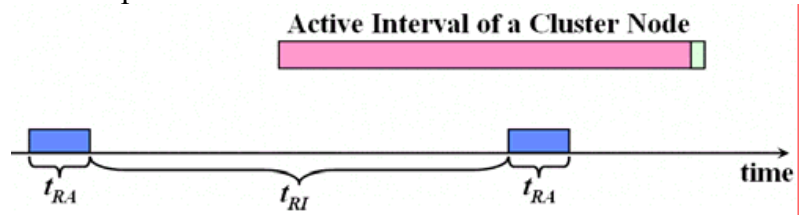

Fig. 5. The resynchronization mechanism

Fig. 5 shows the resynchronization mechanism for the sensor nodes which have no idea about their sleep time. In Fig. 5, a sensor node listens for any packet from its HBG for $t_{R A}$ milliseconds. If it fails, it turns off its antenna to sleep for $t_{R I}$ milliseconds. This sensor node repeats this procedure until it receives a packet whose sender is its HBG. In this situation, it keeps on listen until it receives a packet from its HBG to itself or receives the value of $t_{I}$ broadcasted by its HBG in the ending period. In the first case it can learn its correct sleep time according to the first inner cluster communication protocol. In the second case the sensor node learns the value of $t_{I}$ and then sleeps for $t_{I}$ milliseconds. After it wakes up it should receive the packet from its HBG. In this situation the sensor node keeps listen until it receives a packet from its HBG to itself. This resynchronization mechanism significantly reduces the power consumption for learning the correct active time and sleep time of the sensor nodes.

It should be noted that the values of $t_{R A}$ and $t_{R I}$ decide the performance of the resynchronization mechanism. If $t_{R A}<$ $\max \left\{t_{C I}+t_{C R}, t_{C R}+t_{C S}\right\}$, then the sensor node may capture no complete packet from any member in the same cluster. Because the power consumption of idle listening increases with the value of $t_{R A}$, the smaller value of $t_{R A}$ would reduce more power consumption in idle listening. Therefore, the second inner cluster communication protocol set $t_{R A}=$ $\max \left\{t_{C I}+t_{C R}, t_{C R}+t_{C S}\right\}$.

On the other hand, if $t_{R I}>\max \left\{t_{A}-2 \times t_{R A}, 0\right\}$, then the sensor node may miss the active interval of its cluster node. According to Fig. 5 , it is clear that smaller value of $t_{R I}$ makes the sensor node to try more times before it wakes up in the active interval of its cluster node. This implies that the protocol should try to enlarge the value of $t_{R I}$ to reduce the power consumption in idle listening. However, this increases the possibility that sensor node miss the next active interval of is cluster node and hence enlarges the resynchronization time of the sensor node.

To reduce the resynchronization time of the sensor nodes, the HBG can enlarge the ending period to satisfy the inequality $t_{R I} \leq \max \left\{t_{A}-2 \times t_{R A}, 0\right\}$ and then apply the ending period to repeat the sleep time.

\section{The Inter Cluster Communication Protocols}

\subsection{Adjacent Relationship}

To avoid the interference between adjacent clusters, the HBG should disable the inner communications of the nearby clusters to eliminate the noise from those clusters. Therefore, each HBG should notify all its adjacent HBGs to keep silent in its active interval. Although this idea is reasonable, it brings several problems.

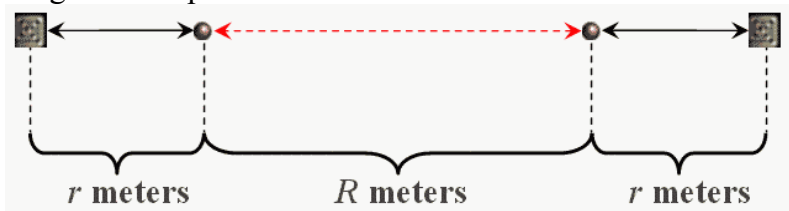

Fig. 6. Interferences between two clusters

The first problem would be the definition of the adjacent relationship. Fig. 6 is helpful in defining an adjacent relationship. In Fig. $6, r$ and $R$ denote the radius of the maximal communication range and interference range of the sensor nodes. Therefore, if the distance between two HBGs is less than $R+2 r$ meters, the communications in one cluster may interfere with the communications in another cluster. Based on this, the first inter cluster communication protocol requires two clusters should not active simultaneously if the distance between their HBGs are less than $R+2 r$ meters.

According to [10], the value of $R$ is greater than $2 r$. Since the received power strength decreases with the square of the distances in single-path free space [11], the first inter cluster communication protocol suggests that the transmitted power for inter cluster communications should be much stronger than 16 times to the transmitted power for inner communications. Besides, this protocol also requires that the bandwidth for inner cluster communications should be distinct from the bandwidth for inter cluster communications. Therefore, each HBG apply at least two different bandwidths. Some for inner cluster communications and the others for inter cluster communications. 


\subsection{Starvation Avoidance}

Applying only the inter cluster communication beacons to disable the adjacent clusters will bring the notorious starvation problem. Consider the scenario in which two patients lie down in adjacent sickbeds. If the values of $t_{A}$ and $t_{I}$ of the two cluster nodes are identical and the two clusters are expected to be active at almost the same time, then the cluster whose active interval is a little latter then the others' may be always disabled by the other.

Most operating systems solve starvation problem by the aging technology [12]. Based on this technology, the second inter cluster communication protocol requires the HBGs to apply the procedure in Fig. 7 to determine their active interval.

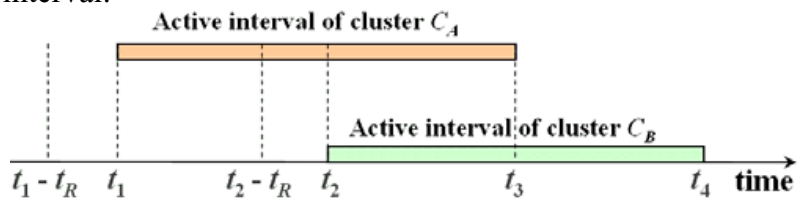

Fig. 7. Competitions of adjacent clusters.

Fig. 7 shows the expected active intervals of two adjacent clusters $C_{A}$ and $C_{B}$. At time $t_{1}-t_{R}$, the HBG of cluster $C_{A}$ broadcasts a packet to all its adjacent HBGs. The packet contains an ordered pair $\left(t_{R}, t_{3}-t_{1}\right)$ and the priority of $C_{A}$. If the priority of $C_{B}$ is less than or equal to the priority of $C_{A}$, then the HBG of $C_{B}$ responses nothing. Otherwise, the HBG of $C_{B}$ sends a packet to the HBG of $C_{A}$ which contains the ordered pair $\left(t_{2}-t_{1}+t_{R}, t_{4}-t_{2}\right)$ before the time $t_{1}$ to disable $C_{A}$ in the interval $\left(t_{2}, t_{3}\right)$.

To solve the starvation problem, the second inter cluster communication protocol apply the following rules to define the priority of each cluster node.

R1. The priority is an integer. 0 is the lowest priority and $M$ is the highest priority.

R2. The initial priority of normal clusters is 0 .

R3. For cluster $C$ whose priority is less than $M-1$, if there is any inner cluster communication of $C$ is disabled by other clusters, the HBG of $C$ increases its priority by 1 .

R4. If all the inner cluster communications of cluster $C$ are not disabled by other clusters, the HBG of cluster $C$ sets its priority as 0 .

R5. If the initial priority of a cluster is $M$, then the HBG does not change its priority.

Since some patients need to be monitored periodically without any disturbance, this protocol reserves the highest priorities to those patients.

\subsection{Exception Handling for Disabled Inner Cluster Communications}

Once an inner cluster communication is disabled, the HBG keeps silent in the corresponding time interval. In this situation, the corresponding sensor node receives no packet from its HBG. According to inner cluster communication protocols, the sensor node will have no idea about its sleep time. According to the inner cluster communication protocols, the sensor node has to apply the resynchronization mechanism to relearn its active and sleep time. Since resynchronization is a costly task and inner cluster communications may be disabled frequently, therefore, the inner cluster communication protocols should provide another resynchronization procedures for the sensor nodes whose inner cluster communications are disabled.

Requiring the sensor nodes to maintain their previous sleep time reveals a possibility of providing a fast and low cost resynchronization procedure. In Fig. 3 and 4, if the value $t_{I}+t_{A}$ is invariant, then the sleep time of each sensor node in the same cluster is also invariant. If the HBG seldom changes the value $t_{I}+t_{A}$, then the next sleep time has high enough possibility to be the same with the previous sleep time. Although applying the previous sleep time is a fast and low cost resynchronization procedure, the scenario in which a sensor node loses the sleep time from its HBG and the new sleep time is different from the previous one still occurs. Therefore, the proposed protocols provide the rules below to resynchronize with the sensor nodes.

R1. Each sensor node maintains a counter $C_{M i s}$ to maintain the times the sensor node receives no packet from its HBG. The initial value of $C_{M i s}$ is 0 and it should be less than a well-known constant $C_{\text {Maxmis }}$.

R2. If a sensor node does not maintain its previous sleep time, it applies the resynchronization procedure in Section 2.3 and set $C_{\text {Mis }}=0$.

R3. If $C_{\text {Mis }}<C_{\text {Maxmis }}-2$ and the sensor node receives no packet from its HBG in $t_{C I}+t_{C R}+t_{C S}$ milliseconds, the sensor node applies the previous sleeps time as its next sleep time and sets $C_{M i s}=C_{M i s}+1$. Otherwise, it applies the resynchronization procedure in Section 2.3 and set $C_{M i s}=0$.

\section{THE HEALTH TELECARE SYSTEM PROTOTYPE}

We have designed and implemented a sensor network prototype for health telecare system. Fig. 8 - Fig. 10 show the sensor node prototypes and the HBG prototype for the health telecare system. The sensor node prototype in Fig. 8 is a wireless clinical thermometer. The RF of this prototype applies the $433 \mathrm{MHz}$ ASK modules. We apply the same RF technology to modify a portable medical appliance in Fig. 9 by adding the RF module. The portable medical appliance contains a sphygmomanometer and a pulsimeter. The HBG can use to attribute "Parameter" to choose the sensor of this sensor node prototype.

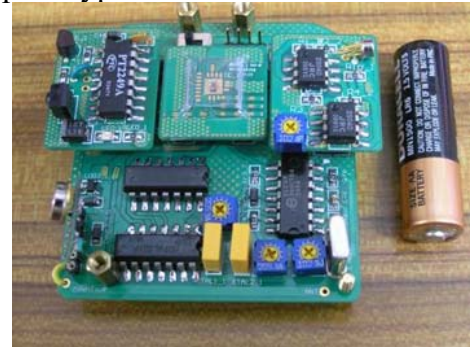

Fig. 8. The wireless clinical thermometer prototype

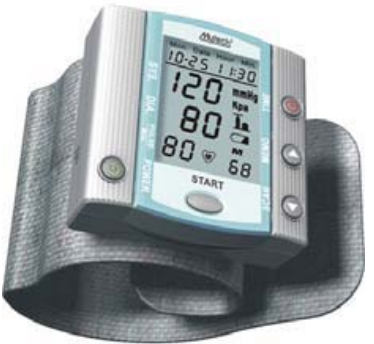

Fig. 9. The wireless sphygmomanometer and pulsimeter prototype 


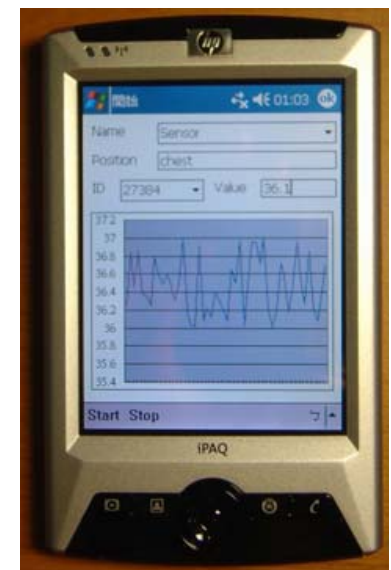

Fig. 10. The HBG prototype

In current stage, we implement the $\mathrm{HBG}$ in a $\mathrm{HP} \mathrm{iPAQ}$ RX3417 PDA. Fig. 10 shows that the HBG presents the past 33 detection results of a clinical thermometer which is detecting the body temperature in the user's chest. The HBG apply its 802.11 WLAN module (in ad hoc mode) to communicate with other HBGs.

\section{DISCUSSIONS}

Although a prototype system based on the proposed technologies has been developed and works well, however, there are still many challenges. Most of the challenges are appropriate parameter specifications for network maintenance. For example, the transmitted power for inner and inter cluster communications, the time interval for $t_{R A}$ and $t_{R I}$ in Fig. 5, the value of $t_{R}$ in Fig. 7, the value of $C_{\text {Maxmis }}$ in Section 3.3, etc. Estimating the appropriate values for those parameters would be a challenge for network maintenance.

Although the proposed inter cluster communication protocols provide priority and aging technology to avoid the inter cluster communication interference problem, there still has at least one exception. Since the clusters are mobile, two non-adjacent clusters which disable no inner clusters communications may become adjacent. In this situation, if the two clusters are still active, then their inner cluster communications may be interfered. In this prototype system, no mechanism is provided for this exception since we think this exception seldom happens. Most exceptions arise from the interferences from other applications such as walky-talky.

\section{CONCLUSIONS AND FUTURE WORKS}

In this paper, we proposed a clustered sensor network architecture for the Wireless $\boldsymbol{H}$ ealth $\boldsymbol{A}$ dvanced Mobile $B$ Bio-diagnostic $S y s t e m$ in long-term periodical health telecare applications. To minimize the power consumptions while reducing the design complexity of the sensor nodes, this study proposes a power saving mechanism and a set of inner cluster communication protocols. Besides, this study also proposes a set of inter cluster communication protocol to avoid most inter cluster interferences.

Although this team has overcame several challenges in implementing a WHAM-BioS prototype, there still are many challenges which obstruct the WHAM-BioS in serving the elders and patients. Scalability is the main challenge for the network functions of the WHAM-BioS. To overcome this challenge, specifying appropriate values for the network parameters would be the first issue. We are now designing simulations and experiments to study the relationships between the parameters and network performance. Besides, to provide a robust platform for health telecare application processes in each HBG, this team is also studying and designing a sensor application platform for the possibly medical applications performing in the HBG.

\section{REFERENCES}

[1]. Tijs van Dam, Koen Langendoen, “An Adaptive Energy-Efficient MAC Protocol for Wireless Sensor Networks," in ACM Sensys., Nov. 2003.

[2]. G. J. Pottie and W. J. Kaiser, "Wireless Integrated Network Sensors," Commun. ACM, vol. 43, no. 5, pp. 51-58, May 2000.

[3]. J. M. Rabaey, et al., "PicoRadio Supports Ad Hoc Ultra-Low Power Wireless Networking," IEEE Computer, vol. 33, no. 7, pp. 42-48, July 2000.

[4]. W. R. Heinzelman, A. Chandrakasan, and H. Balakrishnan, "Energy-Efficient Communication Protocol for Wireless Microsensor Networks," Proc. 33rd Annu. Hawaii Int. Conf. on System Sciences,, pp. $3005-3014,2000$.

[5]. I. F. Akyildiz, W. Su, Y. Sankarasubramaniam, and E. Cayirci, "A Survey on Sensor Networks," IEEE Commun. Mag., pp. 102 - 114, Aug. 2002.

[6]. T. J. Kwon, M. Gerla, V. K. Varma, M. Barton, and T. R. Hsing, "Efficient Flooding with Passive Clustering - An Overhead-Free Selective Forward Mechanism for Ad Hoc/Sensor Networks," IEEE Proceeding, vol. 91, no. 8, pp. 1210-1220, Aug. 2003.

[7]. T. Y. Lin, and Y. C. Tseng, "An Adaptive Sniff Scheduling Scheme for Power Saving in BlueTooth," IEEE Wireless Commun. Mag. pp. 92-103, Dec. 2002.

[8]. Andrew S. Tanenbaum, Computer Networks, Fourth Edition, Prentice-Hall International, INC.

[9]. Ming-Hui Jin, Yu-Cheng Huang, D. Frank Hsu, Cheng-Yan Kao, You-Rui Wu, and Chih-Kung Lee, "On Active Interval Scheduling in Static Sensor Networks," Proceeding of IASTED International Conference on Communication System and Applications, pp. 126 - 131, July 2004..

[10]. D. Dhoutaut and I. Guérin Lassous, "Experiments with 802.11 b in ad hoc configurations," Proceeding of 14th IEEE International Symposium on Personal Indoor and Mobile Radio Communications (PIMRC 2003), pp. 1618-1622, September 2003.

[11]. Kaveh Pahlavan and Allen H. Levesque, Wireless Information Networks, A wiley-Interscience Publication, JOHN WILEY \& SONS, INC.

[12]. Andrew S. Tanenbaum, OPERATING SYSTEM: Design and Implementation, Prentice-Hall International Editions. 